\title{
Is HIDA Scan Necessary for Sonographically Suspicious Cholecystitis?
}

\author{
Irina Bernescu ${ }^{a}$, Oliver S. Enga, b, Lindsay Potdevin ${ }^{a}$, Rosebel Monteiro, \\ Jeffrey Mino ${ }^{a}$, Eric I. Changa ${ }^{\mathrm{a}}$, Tomer Davidov ${ }^{\mathrm{a}}$
}

\begin{abstract}
Background: Historically, abdominal ultrasound is the study of choice for diagnosing cholelithiasis, whereas HIDA scan is the study of choice for diagnosing acute cholecystitis. With improvements in ultrasound technology over the past two decades, we sought to reevaluate the clinical utility of HIDA scan over ultrasound alone in diagnosing cholecystitis.
\end{abstract}

Methods: A retrospective review of 154 patients admitted to our emergency room with suspicion for cholecystitis who underwent abdominal sonography, HIDA scan, and proceeded to cholecystectomy on the same admission was conducted. Results of ultrasound and HIDA scan were compared to the final surgical pathology.

Results: The two groups did not differ with respect to age or gender. HIDA scan had a greater sensitivity, specificity, and accuracy in diagnosing cholecystitis, but the positive predictive value of ultrasound and HIDA scan were similar.

Conclusions: While HIDA scan may be the test of choice for diagnosing cholecystitis, it may be unnecessary in patients with sonographically suspicious cholecystitis, as these sonographic features, when present, are highly predictive of cholecystitis.

Keywords: Cholecystitis; Diagnosis; HIDA; Predictive; Ultrasound

Manuscript accepted for publication September 26, 2013

\footnotetext{
${ }^{a}$ Department of Surgery, Rutgers-Robert Wood Johnson Medical School, New Brunswick, NJ 08903, USA

${ }^{\mathrm{b}}$ Corresponding to: Oliver S. Eng, Department of Surgery, Rutgers-Robert Wood Johnson Medical School, MEB 596, PO Box 19, New Brunswick, NJ 08903, USA. Email: engos@rwjms.rutgers.edu
}

doi: http://dx.doi.org/10.4021/jcs201w

\section{Introduction}

Gallbladder disease is a common problem, particularly in the United States and other western countries $[1,2]$. It is estimated that 20 - 25 million Americans have gallstones, roughly $10-15 \%$ of the adult population [3]. Of these, 10$20 \%$ become symptomatic, often requiring cholecystectomy. Gallbladder disease represents a major health burden, with direct plus indirect costs of approximately $\$ 6.2$ billion annually in the United States [4].

Acute cholecystitis accounts for 3-9\% of hospital admissions for acute abdominal pain [5]. Historically, ultrasound is considered the study of choice for diagnosing cholelithiasis, and HIDA (hepatobiliary iminodiacetic acid) scan is the study of choice for diagnosing acute cholecystitis, given the superior sensitivity and specificity of each test for its respective diagnosis [6]. In the past several decades, there have been technological improvements in ultrasound imaging, resulting in improvements in spatial and contrast resolution [7]. The aim of our study was to reevaluate whether HIDA scan adds to the diagnostic accuracy of contemporary ultrasound alone in patients with suspected cholecystitis.

\section{Materials and Methods}

We performed an IRB-approved retrospective review of patients presenting with presumed cholecystitis between 2007 and 2010, through the Emergency Department at a 600-bed academic medical center. We identified 154 patients who underwent abdominal ultrasound and HIDA scan, and subsequently proceeded to cholecystectomy. Ultrasound results were compared to those of the HIDA scans, using the pathology findings following cholecystectomy as the gold standard for the diagnosis of cholecystitis.

Ultrasounds of the right upper quadrant of the abdomen at our institution were performed using the Philips Healthcare iU22 ultrasound system (Bothell, WA, USA). Images generated included views of the gallbladder from transverse and longitudinal perspectives, identification of gallstones and/or sludge, and measurements of gallbladder wall thick- 
Table 1. Ultrasound Versus HIDA Scan in Diagnosing Cholecystitis

\begin{tabular}{|c|c|c|c|c|c|}
\hline & Sensitivity & Specificity & PPV & NPV & Accuracy \\
\hline Ultrasound & $47 \%$ & $38 \%$ & $96 \%$ & $6 \%$ & $47 \%$ \\
\hline HIDA & $62 \%$ & $50 \%$ & $96 \%$ & $7 \%$ & $62 \%$ \\
\hline$P$ value & 0.009 & 0.029 & 1 & 0.644 & 0.009 \\
\hline
\end{tabular}

ness and diameter of the common bile duct. Ultrasound criteria for acute cholecystitis included: gallbladder wall thickening $3 \mathrm{~mm}$ or greater, the presence of pericholecystic fluid, or a sonographic Murphy's sign.

Acute cholecystitis on HIDA scan was defined as nonvisualization of the gallbladder at four hours. At our institution, a $4 \mathrm{mCi}$ bolus of Tc-99m-mebrofenin was used as the biliary contrast for HIDA scans. If the gallbladder was not visualized by sixty minutes, morphine was administered to hasten visualization of the gallbladder if the cystic duct was not obstructed.

Pathological criteria for the diagnosis of acute cholecystitis included evidence of acute inflammation on macroscopic evaluation, such as congestion, edema, serositis, and fibrin deposition on the gallbladder surface, as well as the microscopic presence of hyperemia, polymorphonuclear neutrophils, and edema. Chronic cholecystitis was diagnosed based on appearance of prior inflammation, including fibrin deposition and wall thickening (in particular, the muscularis propria). Microscopically, the presence of Rokitansky-Aschoff sinusosis, metaplastic epithelial changes, macrophages, lymphocytes, and plasma cells were diagnostic of chronic cholecystitis. The diagnosis of mucosal autolysis was considered a finding associated with bile stasis. Chronic cholecystitis and mucosal autolysis included in our definition of cholecystitis.

We used the pathological diagnosis of cholecystitis to assign each ultrasound and HIDA scan result a true/false and negative/positive value. We then calculated descriptive statistics including sensitivity, specificity, positive predictive value (PPV), negative predictive value (NPV), and accuracy for each of the two tests. P values were obtained using ChiSquared tests.

Table 2. Hospital Cost of Ultrasound Versus HIDA Scan at Our Institution

Ultrasound HIDA

$\begin{array}{lll}\text { Cost, } \$ & 112.57 \quad 342.88\end{array}$

\section{Results}

Patient ages ranged from 16 - 91 years old with an average age of 55 years. Of 154 patients, 44 had evidence of acute cholecystitis, 93 had evidence of chronic cholecystitis, 8 had mucosal autolysis, and 9 had no histologic evidence of any form of cholecystitis. The contingency table and calculated descriptive statistics are in Table 1. While HIDA scan demonstrated a greater sensitivity, specificity, and accuracy in diagnosing cholecystitis compared to ultrasound, the PPV of both were $96 \%(\mathrm{P}=1)$.

\section{Discussion}

Literature has shown that ultrasound has a sensitivity of 84$97 \%$ for diagnosing cholelithiasis [6], and a more variable sensitivity ranging from $48-94 \%$ for diagnosing acute cholecystitis $[6,8,9]$. On the contrary, HIDA scan has a sensitivity of $86-100 \%$ for diagnosing acute cholecystitis [6, 8, 9]. U1trasound has therefore been the study of choice for diagnosing cholelithiasis for the past 3 decades, and HIDA scan has become the study of choice for diagnosing acute cholecystitis. With improvements in ultrasound technology, we sought to re-evaluate whether a HIDA scan is necessary for the patient with acute biliary colic symptoms and an ultrasound that shows gallstones, as well as sonographic features of cholecystitis. We hypothesized that HIDA scan is not necessary, as sonographic features of cholecystitis, when present, are highly predictive of cholecystitis, obviating the need for further imaging. Our study provides an updated comparison of ultrasound vs. HIDA scan, and suggests that ultrasound alone has a PPV of $96 \%$ for diagnosing cholecystits compared to a PPV of $96 \%$ for HIDA scan.

The added cost of a HIDA scan, shown in Table 2, and the delay in definitive early surgical intervention for acute cholecystitis while awaiting HIDA scan should also be considered. Early cholecystectomy has been shown to decrease hospital lengths of stay, complications, and healthcare costs [10-16]. Moreover, the decision to proceed with urgent vs. elective cholecystectomy should be based not only on ul- 
trasound or HIDA findings, but on clinical signs of cholecystitis, such as persistent nausea or pain, severe abdominal tenderness/Murphy's sign, persistent fever, leukocytosis, or frank signs of sepsis.

When considering the results of our study, we acknowledge certain limitations, the first of which would be the retrospective nature of our investigation. Like many similar studies that use surgical findings to confirm diagnoses, our study is affected by verification bias (also called workup bias), introduced by the fact that our gold standard test (cholecystectomy) was performed based on the findings of the tests being evaluated (ultrasound and HIDA). Next, while within the range of reported literature $[6,8,9]$, our sensitivity of diagnosing cholecystitis by ultrasound may have been adversely impacted by the fact that clinical context (fever, leukocytosis, Murphy's sign on physical exam) was not accounted for in our analysis. In addition, the patients selected for this study were initially identified based on having undergone a HIDA scan, prior to researching ultrasound results and operative data. This certainly introduced a selection bias, as many patients who present with definitive ultrasound findings of acute cholecystitis in an adequate clinical context may not then undergo a HIDA scan.

Despite these limitations, our study indicates that HIDA scan is still superior to ultrasound in diagnosing cholecystitis, having a higher sensitivity, specificity, and accuracy than ultrasound, but with a similar PPV. Given the increasing availability of ultrasound imaging, as well as the increasing proficiency of medical personnel in performing this diagnostic test, ultrasound has the potential of becoming a true extension of the physical exam, rendering other tests unnecessary in the diagnosis of specific conditions, such as cholecystitis. Finally, such findings are particularly relevant and timely in the era of increased awareness to healthcare costs.

\section{Sources of Funding}

None

\section{Conflict of Interest}

None declared.

\section{Financial Disclosures}

The authors declare that they have no financial disclosures.

\section{References}

1. Angelico F, Del Ben M, Barbato A, Conti R, Urbinati G.
Ten-year incidence and natural history of gallstone disease in a rural population of women in central Italy. The Rome Group for the Epidemiology and Prevention of Cholelithiasis (GREPCO). Ital J Gastroenterol Hepatol. 1997;29(3):249-254.

2. Jensen $\mathrm{KH}$, Jorgensen $\mathrm{T}$. Incidence of gallstones in a Danish population. Gastroenterology. 1991;100(3):790794.

3. Shaffer EA. Gallstone disease: Epidemiology of gallbladder stone disease. Best Pract Res Clin Gastroenterol. 2006;20(6):981-996.

4. Everhart JE, Ruhl CE. Burden of digestive diseases in the United States part I: overall and upper gastrointestinal diseases. Gastroenterology. 2009;136(2):376-386.

5. Trowbridge RL, Rutkowski NK, Shojania KG. Does this patient have acute cholecystitis? JAMA. 2003;289(1):8086.

6. Shea JA, Berlin JA, Escarce JJ, Clarke JR, Kinosian BP, Cabana MD, Tsai WW, et al. Revised estimates of diagnostic test sensitivity and specificity in suspected biliary tract disease. Arch Intern Med. 1994;154(22):2573-2581.

7. Harvey CJ, Pilcher JM, Eckersley RJ, Blomley MJ, Cosgrove DO. Advances in ultrasound. Clin Radiol. 2002;57(3):157-177.

8. Alobaidi M, Gupta R, Jafri SZ, Fink-Bennet DM. Current trends in imaging evaluation of acute cholecystitis. Emerg Radiol. 2004;10(5):256-258.

9. Kalimi R, Gecelter GR, Caplin D, Brickman M, Tronco GT, Love C, Yao J, et al. Diagnosis of acute cholecystitis: sensitivity of sonography, cholescintigraphy, and combined sonography-cholescintigraphy. J Am Coll Surg. 2001;193(6):609-613.

10. Casillas RA, Yegiyants S, Collins JC. Early laparoscopic cholecystectomy is the preferred management of acute cholecystitis. Arch Surg. 2008;143(6):533-537.

11. Chang TC, Lin MT, Wu MH, Wang MY, Lee PH. Evaluation of early versus delayed laparoscopic cholecystectomy in the treatment of acute cholecystitis. Hepatogastroenterology. 2009;56(89):26-28.

12. Gonzalez-Rodriguez FJ, Paredes-Cotore JP, Ponton C, Rojo Y, Flores E, Luis-Calo ES, Barreiro-Morandeira F, et al. Early or delayed laparoscopic cholecystectomy in acute cholecystitis? Conclusions of a controlled trial. Hepatogastroenterology. 2009;56(89):11-16.

13. Lau B, Difronzo LA. An acute care surgery model improves timeliness of care and reduces hospital stay for patients with acute cholecystitis. Am Surg. 2011;77(10):1318-1321.

14. Lo CM, Liu CL, Fan ST, Lai EC, Wong J. Prospective randomized study of early versus delayed laparoscopic cholecystectomy for acute cholecystitis. Ann Surg. 1998;227(4):461-467.

15. Gurusamy K, Samraj K, Gluud C, Wilson E, Davidson BR. Meta-analysis of randomized controlled trials 
on the safety and effectiveness of early versus delayed laparoscopic cholecystectomy for acute cholecystitis. $\mathrm{Br}$ J Surg. 2010;97(2):141-150.
16. Johner A, Raymakers A, Wiseman SM. Cost utility of early versus delayed laparoscopic cholecystectomy for acute cholecystitis. Surg Endosc. 2013;27(1):256-262. 